\title{
Amassing Granular Information About Eosinophilic Gastrointestinal Disorders Through Multicenter Cooperation: A Paradigm for Rare Disease Study
}

\author{
Anupama Ravi ${ }^{1}$. David A. Katzka ${ }^{1}$
}

Published online: 22 January 2020

(c) Springer Science+Business Media, LLC, part of Springer Nature 2020

Eosinophilic gastrointestinal disorders (EGIDs) represent a group of diseases in which abnormal infiltration of the gut layers with eosinophils manifests with signs of bowel inflammation and/or obstruction, malabsorption, failure to thrive, and bleeding. These disorders vary by location (gastric, small bowel and/or colon), gut layer involvement (mucosa, submucosa, muscle and/or serosa), association with peripheral eosinophilia, and degree of systemic involvement. They may overlap with eosinophilic esophagitis in $10 \%$ of patients, particularly when the stomach and/or small bowel is involved [1]. They are distinguished from the hypereosinophilic syndromes, which are a group of systemic disorders marked primarily by robust peripheral eosinophilia secondary to clonal expansion or IL-5 mediated proliferation of eosinophils. Multiple treatments have been proposed for EGIDs including dietary therapy, topical and systemic steroids, immunosuppressive agents, and biologics.

One of the difficulties in better characterizing and treating EGIDs is their relative rarity. With an estimated prevalence of 2.1-5.1 per 100,000 [1,2], most of the experience is derived from patient series from single academic centers. To overcome this limitation, the Consortium of Eosinophilic Gastrointestinal Researchers (CEGIR) was established, a consortium comprised of investigators from academic medical centers, a part of the NIH Rare Diseases Clinical Research Network, with a multidisciplinary adult and pediatric interest in EGIDs that was formed for the purpose of combining patient data about this rare group of diseases to better study its characteristics and treatment.

The initial publication from this group reported that the rate of diagnosis of EGIDs has been increasing with a

David A. Katzka

katzka.david@mayo.edu

1 Division of Gastroenterology and Hepatology (DAK), Department of Pediatrics (AR), Mayo 9, Mayo Clinic, 200 First St., S.W., Rochester, MN 55905, USA slight predominance of eosinophilic gastritis over that of enteritis and colitis [3]. More than a third of patients had eosinophilic inflammation outside of their primary disease location, most commonly within the esophagus. In this issue of Digestive Diseases and Sciences, Pesek along with multiple members of the CEGIR extends these results by more intensively studying the endoscopic and histologic characteristics of these diseases [4]. The consortium reports that the endoscopic appearance of gut segments is normal in most patients, prompting biopsy of these areas if there is a suspicion for EGID. Visualized abnormalities, including nodularity, ulceration, erythema, and mucosal friability, were non-diagnostic. Thus, biopsies should be obtained whenever EGIDs are suspected. Regarding histology, although eosinophil density could be markedly elevated (500 eosinophils per high power field), the distribution was often patchy, indicating the need to obtain multiple biopsies at multiple locations. In fact, some grossly involved organs commonly had normal eosinophil density. In addition to tissue eosinophilia, a plethora of other abnormalities could be seen, including eosinophil degranulation, lymphocytosis, cryptitis, crypt abscesses, and lamina propria or muscularis involvement. Notably however, none of these abnormalities dominated the histologic picture, underscoring the need to make this diagnosis through comprehensive evaluation and sampling of the gastrointestinal tract. Finally, although multiple therapies were used among the several centers, there was generally, although not uniformly, a good correlation between symptomatic relief and reductions in tissue eosinophilia and endoscopic abnormalities. It remains to be determined whether any of these objective measures will be important in monitoring disease and whether the concept of deep histologic or endoscopic remission, as in inflammatory bowel disease, will become the therapeutic goal. More likely, as with eosinophilic esophagitis, patient response will be assessed through a combination of tools. 
Despite these advances, many diagnostic and therapeutic questions remain. The threshold density for tissue eosinophilia is still somewhat arbitrary both in absolute count and uniformity within organ biopsies. Is there a non-invasive marker for tissue involvement? Is there a standardized definition or criteria for diagnosing EGIDs? What is the best therapy, inhibitors of IL-5 [5] such as mepolizumab or benralizumab or IgE inhibitors [6] such as omalizumab?

Fortunately, this study reinforces the validity of the core tenets of EGID: tissue eosinophilia must be present, and other causes of tissue eosinophilia such as parasitic disease, inflammatory bowel disease, or malignancy must be excluded. The work from CEGIR not only confirms what is known but also begins the process of standardizing the EGIDs knowledge base. The potential of this important consortium is formidable not only for gathering additional information on these diseases but perhaps even for informing the rationale for, the design of, and the conduct of clinical trials. For those readers who might still dismiss this study as one addressing a rare eosinophilic disease not relevant to practice, the experience with eosinophilic esophagitis is instructive, edifying, and enlightening.

\section{Compliance with Ethical Standards}

Conflict of interest DAK: Honorarium from Celgene for lecture at their facilities, 2019. AR: The author declares that she has no conflict of interest.

\section{References}

1. Jensen ET, Martin CF, Kappelman MD, Dellon ES. Prevalence of eosinophilic gastritis, gastroenteritis, and colitis: estimates from a national administrative database. J Pediatr Gastroenterol Nutr. 2016;62:36-42.

2. Moawad FJ. Eosinophilic esophagitis: incidence and prevalence. Gastrointest Endosc Clin N Am. 2018;28:15-25.

3. Pesek RD, Reed CC, Muir AB, et al. Increasing rates of diagnosis, substantial co-occurrence, and variable treatment patterns of eosinophilic gastritis, gastroenteritis, and colitis based on 10 -year data across a multicenter consortium. Am J Gastroenterol. 2019;114:984-994.

4. Pesek RD, Reed CC, Collins MH, et al. (2019) Association between endoscopic and histologic findings in a multi-center retrospective cohort of patients with non-esophageal eosinophilic gastrointestinal disorders. Dig Dis Sci. (Epub ahead of print). https ://doi.org/10.1007/s10620-019-05961-4.

5. Kim YJ, Prussin C, Martin B, et al. Rebound eosinophilia after treatment of hypereosinophilic syndrome and eosinophilic gastroenteritis with monoclonal anti-IL-5 antibody SCH55700. $J$ Allergy Clin Immunol. 2004;114:1449-1455.

6. Foroughi S, Foster B, Kim N, et al. Anti-IgE treatment of eosinophil-associated gastrointestinal disorders. J Allergy Clin Immunol. 2007;120:594-601.

Publisher's Note Springer Nature remains neutral with regard to jurisdictional claims in published maps and institutional affiliations. 